\title{
Genetic Variability and Trait Association Analysis for Agro-Morphological Markers in Mulberry Genetic Resources from Kashmir, India
}

\author{
Pawan Saini $^{*}$, S.S. Chauhan ${ }^{1}$, Aftab A. Shabnam ${ }^{1}$, \\ Lal Chand ${ }^{2}$ and Narender $\mathrm{Negi}^{3}$ \\ ${ }^{1}$ CSB-Central Sericultural Research and Training Institute, Pampore-192121, \\ Jammu and Kashmir, India \\ ${ }^{2}$ ICAR-Central Agro-forestry Research Institute, Jhansi-284003, Uttar Pradesh, India \\ ${ }^{3}$ ICAR-Regional Station, National Bureau of Plant Genetic Resources, Shimla, \\ Himachal Pradesh, India \\ *Corresponding author
}

\section{A B S T R A C T}

Genetic variability is the pre-requisite for the initiation of any improvement programme for the identification and selection of superior entries over the existing cultivars. The

\begin{tabular}{|l|}
\hline Ke y w or d s \\
$\begin{array}{l}\text { Character association } \\
\text { analysis, Genetic } \\
\text { resources, Heritability, } \\
\text { Mulberry, Selection }\end{array}$ \\
\hline Article Info \\
\hline $\begin{array}{l}\text { Accepted: } \\
16 \text { March } 2018 \\
\text { Available Online: } \\
10 \text { April } 2018\end{array}$ \\
\hline \hline
\end{tabular}
investigation was conducted in 47 genotypes maintained at mulberry germplasm block at CSB-Central Sericultural Research and Training Institute (CSRandTI), Pampore for 13 agro-morphological traits to understand the available genetic variability for future improvement in mulberry. Analysis of variance (ANOVA) showed highly significant differences $(\mathrm{P}=0.01)$ between the genotypes for the agro-morphological traits studied. High level of phenotypic and genotypic coefficient of variation $(>20 \%)$ observed for petiole length, petiole weight, leaf length, leaf width, number of leaf attached on main shoot, number of nodes on main shoot, total shoot length, leaf weight of main shoot, main shoot weight, total shoot weight per plant and total leaf weight or leaf yield indicated that these traits are governed by genetic factors. High heritability estimates coupled with high genetic advance as per cent of mean (GAM) for indicated additive gene action and improvement can be made through selection. Correlation coefficient association analysis revealed significant and positive correlation of leaf yield with yield components. The study revealed importance of direct selection for the improvement of agro-morphological traits.

\section{Introduction}

Mulberry is a fast growing deciduous, perennial and highly heterozygous plant which exhibit sexual polymorphism. It is believed to have originated in the Northern hemisphere, particularly in the Himalayan foothills and spreads to the tropics of Southern hemisphere
(Benavidas et al., 1994). It can be grown in diverse edapho-climatic conditions which require more productive hybrids for acclimatization in particular area (Tikader $e t$ al., 2004). It is the primary food plant of silkworm (Bombyx mori L.); hence, availability of good quality leaf has great impact on the sustainability and profitability 
of sericulture industry (Vijayan et al., 2010). Increased production of silk depends, to a great extent on increased leaf yield of mulberry plant (Sarkar et al., 1987). The genetic resources could enable development of cultivars not only with improved productivity but also biotic and abiotic stress tolerance (Tanksley and McCouch, 1997). Because of wide behavioral variation and easily adapted to varying ecological conditions, mulberry easily hybridized both naturally as well as artificially which creates a wide range of variability in the existing gene pool (Zhao et al., 2006). The extent of magnitude of genetic variability in the mulberry germplasm helps in the crop improvement through conventional breeding. Genetic variability is the prerequisite for initiation of any crop improvement programme including mulberry and selection acts upon the variability which is present in the genotypes. For making effective selection basing upon the metric traits estimation of genetic variability parameters heritability and genetic advance as per percent of mean (GAM) indicates the extent of trait transmissibility generation to generation.

Trait association analysis draws a clear image of inter-relationships and relative contribution of independent variables on dependent variables, which enables a plant breeder to make selection procedures for crop improvement (Dewey and Lu, 1959 and Bhat, 1973). Genetic variability and trait association studies for various agro-morphological traits in mulberry has been reported by various researchers in India (Sarkar et al., 1987; Bari et al., 1989; Susheelamma et al., 1998; Goel et al., 1998; Vijayan et al., 1998; Masilamani et al., 2000; Tikader and Roy, 2001; Tikader et al., 2004; Tikader and Dandin, 2005; Rahman et al., 2006; Doss et al., 2006; Banerjee et al., 2007; Mallikarjunnappa et al., 2008; Vijayan et al., 2010; Doss et al., 2011; Doss et al., 2012; Biradar et al., 2015 and Suresh et al., 2017). The North-Western Himalayan region of India is gifted with very rich diversity of mulberry with high morpho-genetic variability. Characterization and evaluation of diverse genotypes is a continuous process for improvement in terms of yield, quality and tolerance to biotic and abiotic stress to evolve new varieties / hybrids for diverse agroclimatic regions. Central Silk Board - Central Sericultural Research and Training Institute, Pampore has a collection of temperate indigenous and exotic mulberry genotypes representing five countries. Hence, the present study was conducted with the objective of characterization of mulberry genotypes and to mine genetic variability among 47 mulberry genotypes conserved in the field gene bank of CSB-CSRandTI, Pampore to develop good quality and high leaf yielding varieties / hybrids suitable to highly variable agroclimatic regions.

\section{Materials and Methods}

\section{Experimental site and environment}

The present study was conducted at the Mulberry Germplasm Block, CSB-CSRandTI, Pampore. The institute is situated at $34^{0} 02^{\prime} \mathrm{N}$ latitude and $74^{0} 93$ ' $\mathrm{E}$ longitude and at an altitude of $1573 \mathrm{~m}$ above mean sea level. The soil type is clay loam. The bio-climate of Kashmir valley is dry temperate and humid. The annual rainfall is $635 \mathrm{~mm}$ and the average winter and summer temperature is 2.5 and $24.1^{\circ} \mathrm{C}$.

\section{Experimental material}

The experimental material comprised of 47 mulberry genotypes (16 indigenous and 31 exotic) (Table 1). In April, 2015 the 47 genotypes were planted at spacing of $90 \times 180$ $\mathrm{cm}$ at mulberry germplasm block of the institute and managed by following the recommended agronomic package of practices (Ahsan et al., 1990). 


\section{Experimental data}

Quantitative traits like petiole length $(\mathrm{cm})$, petiole weight $(\mathrm{gm})$, leaf length $(\mathrm{cm})$, leaf width $(\mathrm{cm})$, number of leaf attached on main shoot, number of nodes on main shoot, total number of shoots per plant, length of longest shoot $(\mathrm{cm})$, total shoot length $(\mathrm{cm})$, leaf weight of main shoot $(\mathrm{kg})$, main shoot weight $(\mathrm{kg})$, total shoot weight per plant $(\mathrm{kg})$ and total leaf weight or leaf yield $(\mathrm{kg})$ were recorded from randomly sampled three replications. The traits were recorded as per the DUS descriptors developed by Central Sericultural Research and Training Institute, Mysore (Sivaprasad et al., 2016).

\section{Statistical analysis and estimation of genetic parameters}

The mean data of the above mentioned traits were statistically analyzed, using the standard method suggested by Clewer and Scarisbrick (2001), using the Windostat version 9.2 package program. Analysis of variance (ANOVA) was done by the method suggested by Panse and Sukhatme (1985). The phenotypic coefficient of variation (PCV), genotypic coefficient of variation (GCV) was estimated as suggested by Burton and De vane (1953). Heritability and genetic gain were calculated by following Lush et al., (1945) and Johnson et al., (1955) respectively. The correlation coefficient analysis among all the possible combination at phenotypic (rp) and genotypic (rg) level were estimated employing the formulae (Al-Jibourie et al., 1958). Significance of correlation coefficient at both phenotypic and genotypic levels was tested by comparing table ' $r$ ' value with the obtained value. The Path coefficient is a standardized partial regression coefficient and as such it is a measure of direct and indirect effect of a set variable (component characters) as a dependent variable such as leaf yield. The estimates of direct and indirect effect of component characters on leaf yield were computed using appropriate correlation coefficient of different component characters as suggested by Wright (1921) and elaborated by Dewey and Lu (1959). Thus, the correlation coefficient of any character/trait with leaf yield was split into direct and indirect effects adopting the standard formula.

\section{Results and Discussion}

Genetic variability, heritability and genetic advance

For any genetic improvement programme in crop plants, the availability of large genetic stocks representing diverse genotypes is a pre requisite. In addition to maintaining the pure stocks of the entries, it is also essential to make a systematic assessment of the extent of variability present for various yield components for effective selection of genotypes to bring about improvement in the desired direction.

The analysis of variance among forty seven (47) genotypes of mulberry indicated highly significant differences among them for thirteen (13) agro-morphological traits indicating presence of sufficient amount of variability in respect of all the traits studied (Table 2). The genotypic differences were significant at $\mathrm{P}=0.01$. Similar results are reported by Sarkar et al., (1987), Tikader and Roy (2001), Tikader et al., (2004), Doss et al., (2006), Banerjee et al., (2007), Mallikarjunappa et al., (2008), Vijayashekara (2009) and Biradar et al., (2015).

The estimates of range, population mean, variance and genetic parameters viz., phenotypic, genotypic and environmental coefficient of variation, heritability (broad sense) and genetic advance for nut and kernel traits are presented in Table 3 and Figure 1. 
The range in mean values does not reflect the total variance in the traits studied amongst all the genotypes. There are large differences observed between the minimum and maximum range. Hence, actual variance has to be estimated for the characters to know the extent of existing variability. The genotypic variance measures the magnitude of genetic variability present in the crop and phenotypic variance indicates the amount of variation which is due to the phenotypic values. The estimated phenotypic variance for all the traits was higher than genotypic variance. Similar kinds of results were also reported by Mallaikarjunappa et al., (2008) and Suresh et al., (2017). The overall coefficient of variation (CV) value ranges 9.79-23.71. The wide range of variation obtained may be due to divergent genotypes included in the study. The presence of such wide variability in mulberry with respect to all the traits indicating that significant variation existed among the genotypes.

The phenotypic coefficient of variation (PCV) was also found to be higher than genotypic coefficient of variation (GCV). Leaf yield is a polygenic trait which is highly influenced by the environmental factors. The phenotypic / observable variation is the combined effect of genetic factors as well as environmental factors. High level of phenotypic and genotypic coefficient of variation $(>20 \%)$ was observed for petiole length, petiole weight, leaf length, leaf width, number of leaf attached on main shoot, number of nodes on main shoot, total shoot length, leaf weight of main shoot, main shoot weight, total shoot weight per plant and total leaf weight or leaf yield indicated that these traits are governed by genetic factors and existence greater magnitude of genetic variability among the genotypes and selection will be rewarded for the improvement of these traits. While high PCV $(>20 \%)$ and moderate GCV (10.1$20.0 \%$ ) was recorded for total number of shoots and length of longest shoot indicated high influence of environment than genetic factors and selection for these traits will be less effective. These result are in agree with the observation made by Goel et al., (1998), Tikader et al., (2004), Banerjee et al., (2007), Tikader and Kamble (2008a), Mallikarjunappa et al., (2008), Doss et al., (2012), Biradar et al., (2015) and Suresh et al., (2017).

The selection efficiency was higher when the parameters had higher heritability. The heritability estimates (broad sense) was ranged from $47-93 \%$ and it is high for all the traits studied (Tikader et al., 2004; Banerjee et al., 2007; Biradar et al., 2015 and Suresh et al., 2017) except total number of shoots exhibit moderate level of heritability suggesting additive gene effects and indicated high rate of trait transmissibility into the future generations. Hence, improvement can be made by simple selection. High heritability estimates suggested the major role of genetic constitution in the expression of the characters and such characters are considered to be dependable from the breeding point of view. High heritability coupled with high genetic gain was observed for leaf weight/plant (Goel et al., 1998); lamina weight, 100 leaf weight, number of shoots, petiole weight, total shoot length and leaf yield per plant (Das and Krishnaswamy, 1969; Tikader, 1997 and Tikader et al., 2004); nodal distance, total shoot length, leaf/twig ratio, weight of 100 fresh and dry leaves, single leaf area and leaf yield (Doss et al., 2006), lamina weight, single leaf area and fresh leaf weight (Banerjee et al., 2007); fresh weight of 100 leaves (Mallikarjunappa et al., 2008); number of branches per plant, leaf yield per plant, leafshoot ratio, hundred leaf weight and total shoot length (Keshava Murthy et al., 2010); total shoot length, number of leaves per plant, single leaf area, leaf yield per plant, plant height and weight of 100 fresh leaves (Biradar et al., 2015) and total chlorophyll, specific leaf area, single leaf area, petiole weight, single lamina weight, total shoot length, plant height, 
shoots per plant, average shoot length, leaf yield, shoot yield, intermodal distance and harvest index (Suresh et al., 2017).

A character with high heritability and high genetic gain may be due to additive gene action (Panse, 1957) in the expression of these traits and effective progress in improvement through selection could be achieved for leaf yield. The parameters without such combination may appear because of nonadditive gene action, including dominance and epistasis (Liang and Walter, 1968). It would be worthwhile to resort to breeding methodologies other than conventional pedigree or backcross techniques as these would leave the non-fixable component unexploited. Hence, improvement of agromorphological traits would be effective through phenotypic selection.

\section{Trait-association studies}

\section{Correlation analysis}

Correlation among the 13 yield attributing characters revealed substantial differences between phenotypic and genotypic correlations (table 4). Significant correlation of characters suggested that there is much scope for direct and indirect selection for further improvement. Genotypic correlation coefficient provides measures of genetic association between traits and helps to identify the more important as well as less important traits to be considered in breeding program (Tiwari and Upadhyay, 2011). The magnitude of genotypic correlations was higher than their corresponding phenotypic correlations. This can be interpreted as a strong inherent genotypic relationship between characters studied, though their phenotypic expression was impeded by environmental factors. The present findings are in conformity with Harer et al., (2003), Kumar et al., (2003), Golani et al., (2007), Chikkalingaiah et al., (2009),
Islam et al., (2010), Dar et al., (2011), AlAyesh et al., (2012), Souza et al., (2012) and Tasisa et al., (2012).

In the present investigation, leaf yield is positively and significantly correlated with petiole length, number of leaf attached, total number of shoots, length of longest shoot, total shoot length, leaf weight, shoot weight and total shoot weight at both phenotypic and genotypic level. Similarly, Sarkar et al., (1987), Bari et al., (1989), Vijayan et al., (1997b and 1998), Tikader and Roy (1999 and 2001), Tikader and Dandin (2005), Rahman et al., (2006), Banerjee et al., (2007), Mallikarjunnappa et al., (2008), Doss et al., (2012), Biradar et al., (2015) and Suresh et al., (2017) reported leaf yield association with other quantitative traits in mulberry.

Length of longest shoot shows positive and significant correlation with total shoot length, leaf weight, shoot weight, total shoot weight and leaf yield. Similar observation were also made by Sarkar et al., (1987), Vijayan et al., (1997), Tikader and Roy (2001), Tikader and Dandin (2005), Banerjee et al., (2007) and Birader et al., (2015). Since, in sericulture mulberry leaf productivity is a multifactorial trait which depends upon a number of quantitative traits like plant height, number of shoots, length of shoot, leaf size and weight, moisture retention capacity, total biomass, the association between these traits appears to be reasonable that improvement in these traits through selection will enhance the leaf productivity which will have great impact on sericulture industry.

\section{Path coefficient analysis}

The relationship between growth parameters may be negative or positive but it is the net result of that particular trait and indirect effect via other traits.

Table.1 List of temperate mulberry genotypes present at CSRandTI, Pampore 
Int.J.Curr.Microbiol.App.Sci (2018) 7(4): 1799-1812

\begin{tabular}{|c|c|c|c|c|}
\hline SI. No. & Variety & Species & Indigenous & Exotics \\
\hline 1. & BC-259 & M. alba & Indigenous & \\
\hline 2. & Botatul & M. alba & Indigenous & \\
\hline 3. & Brentul Kashmir & M. alba & Indigenous & \\
\hline 4. & C-4 & M. alba & Indigenous & \\
\hline 5. & C-763 & M. alba & Indigenous & \\
\hline 6. & C-776 & & Indigenous & \\
\hline 7. & C-1733 & M. alba & Indigenous & \\
\hline 8. & Chinese white & M. alba & & China \\
\hline 9. & Enshatakosuke & M. bombycis & & Japan \\
\hline 10. & French & M. alba & & France \\
\hline 11. & Goshoerami & M. multicaulis & & Japan \\
\hline 12. & Himachal local & M. indica & Indigenous & \\
\hline 13. & Ichinose & M. alba & & Japan \\
\hline 14. & Kasuga & M. multicaulis & & Japan \\
\hline 15. & Kanva-2 & & Indigenous & \\
\hline 16. & Kokusou-27 & M. alba & & Japan \\
\hline 17. & Lajward & & Indigenous & \\
\hline 18. & Mandalay (S-1) & M. alba & & Burma \\
\hline 19. & Mysore local & M. indica & Indigenous & \\
\hline 20. & Obawase & M. bombycis & & Japan \\
\hline 21. & Punjab local & M. alba & Indigenous & \\
\hline 22. & Rokokuyaso & M. multicaulis & & Japan \\
\hline 23. & S-36 & & Indigenous & \\
\hline 24. & S-41 & M. alba & Indigenous & \\
\hline 25. & S-54 & & Indigenous & \\
\hline 26. & S-146 & M. alba & Indigenous & \\
\hline 27. & S-799 & M. alba & Indigenous & \\
\hline 28. & S-1301 & M. alba & Indigenous & \\
\hline 29. & S-1531 & M. alba & Indigenous & \\
\hline 30. & S-1635 & M. alba & Indigenous & \\
\hline 31. & S-1708 & M. alba & Indigenous & \\
\hline 32. & $\mathrm{~T}-4$ & M. alba & Indigenous & \\
\hline 33. & $\mathrm{~T}-10$ & M. alba & Indigenous & \\
\hline 34. & Tomeiso & M. alba & & Japan \\
\hline 35. & $\operatorname{Tr}-10$ & M. alba & Indigenous & \\
\hline 36. & Zagtul & M. alba & Indigenous & \\
\hline 37. & AR-14 & M. alba & Indigenous & \\
\hline 38. & BR-2 & M. alba & Indigenous & \\
\hline 39. & Okinowa & Unknown & & Japan \\
\hline 40. & V1 & & Indigenous & \\
\hline 41. & English Black & & & France \\
\hline 42. & K2 x Kosen & Unknown & Indigenous & \\
\hline 43. & ME-27 & & & Exotic \\
\hline 44. & ME-53 & & & Exotic \\
\hline 45. & ME-58 & & & Exotic \\
\hline 46. & Almora Local & & Indigenous & \\
\hline 47. & S-140 & & Indigenous & \\
\hline
\end{tabular}


Table.2 Analysis of variance for yield attributing biometric traits in temperate mulberry germplasm accessions of Jammu and Kashmir

\begin{tabular}{|c|c|c|c|c|c|c|c|c|c|c|c|c|c|c|}
\hline $\begin{array}{l}\text { Source of } \\
\text { variation }\end{array}$ & Df & $\begin{array}{c}\text { Petiole } \\
\text { Length } \\
(\mathrm{cm})\end{array}$ & $\begin{array}{l}\text { Petiole } \\
\text { Weight } \\
(\mathrm{gm})\end{array}$ & $\begin{array}{c}\text { Leaf } \\
\text { Length } \\
(\mathrm{cm})\end{array}$ & $\begin{array}{l}\text { Leaf } \\
\text { Width } \\
(\mathrm{cm})\end{array}$ & $\begin{array}{c}\text { Number of leaf } \\
\text { attached }\end{array}$ & $\begin{array}{l}\text { Number of } \\
\text { nodes }\end{array}$ & $\begin{array}{c}\text { Total } \\
\text { number } \\
\text { of shoots }\end{array}$ & $\begin{array}{l}\text { Length of } \\
\text { longest shoot } \\
\text { (cm) }\end{array}$ & $\begin{array}{l}\text { Total Shoot } \\
\text { length }(\mathrm{cm})\end{array}$ & $\begin{array}{c}\text { Leaf } \\
\text { Weight } \\
(\mathrm{kg})\end{array}$ & $\begin{array}{c}\text { Shoot } \\
\text { weight } \\
(\mathbf{k g})\end{array}$ & $\begin{array}{l}\text { Total shoot } \\
\text { weight }(\mathrm{kg})\end{array}$ & $\begin{array}{l}\text { Leaf } \\
\text { Yield } \\
(\mathrm{Kg})\end{array}$ \\
\hline Replication & 2 & 0.6854 & 0.2466 & 0.3141 & 5.9313 & 228.0976 & 60.3688 & 0.6436 & 66.1489 & 8419.2139 & 0.0020 & 0.0007 & 0.0181 & 1.1571 \\
\hline Treatment & 46 & $3.4385 * *$ & $5.8768^{* *}$ & $32.8055^{* *}$ & $23.0879 * *$ & $36882.9570 * *$ & $1137.9948^{* *}$ & $3.4588^{* *}$ & $1871.5785^{* *}$ & $111964.4922 * *$ & $0.3996^{* *}$ & $0.9802 * *$ & $9.6256^{* *}$ & $6.8373^{* *}$ \\
\hline Error & 92 & 0.3857 & 0.4165 & 2.8909 & 3.1308 & 887.3910 & 28.7583 & 0.9407 & 140.7866 & 10919.3145 & 0.0153 & 0.0542 & 0.3771 & 0.5240 \\
\hline
\end{tabular}

Table.3 Genetic parameters for yield attributing biometric traits in temperate mulberry germplasm accessions of Jammu and Kashmir

\begin{tabular}{|c|c|c|c|c|c|c|c|c|c|c|c|c|c|c|}
\hline \multirow{2}{*}{$\begin{array}{l}\text { SI. } \\
\text { No. }\end{array}$} & \multirow[t]{2}{*}{ Traits } & \multirow[t]{2}{*}{ Mean \pm SE } & \multicolumn{2}{|c|}{ Range } & \multicolumn{3}{|c|}{ Variance } & \multicolumn{4}{|c|}{ Coefficient of Variability } & \multirow{2}{*}{$\begin{array}{c}\mathbf{H}^{2} \\
\text { (Broad } \\
\text { sense) } \\
(\%)\end{array}$} & \multirow{2}{*}{$\begin{array}{c}\text { Genetic } \\
\text { Advance } \\
\text { (GA) }\end{array}$} & \multirow{2}{*}{$\begin{array}{l}\text { GA as per } \\
\text { cent of } \\
\text { means }\end{array}$} \\
\hline & & & Minimum & Maximum & PV & GV & $\mathbf{E V}$ & $\begin{array}{l}\text { General } \\
\text { CV }(\%)\end{array}$ & $\begin{array}{l}\text { PCV } \\
(\%)\end{array}$ & $\begin{array}{l}\text { GCV } \\
(\%)\end{array}$ & $\begin{array}{l}\text { ECV } \\
(\%)\end{array}$ & & & \\
\hline 1 & Petiole length (cm) & $4.22 \pm 0.36$ & 2.40 & 6.83 & 1.40 & 1.02 & 0.39 & 14.72 & 28.09 & 23.92 & 14.72 & 0.73 & 1.77 & 41.96 \\
\hline 2 & Petiole weight (gm) & $2.96 \pm 0.37$ & 0.77 & 6.50 & 2.24 & 1.82 & 0.42 & 21.82 & 50.57 & 45.62 & 21.82 & 0.81 & 2.51 & 84.77 \\
\hline 3 & Leaf length (cm) & $14.50 \pm 0.98$ & 8.77 & 24.33 & 12.86 & 9.97 & 2.89 & 11.73 & 24.74 & 21.78 & 11.73 & 0.78 & 5.73 & 39.51 \\
\hline 4 & Leaf width (cm) & $11.11 \pm 1.02$ & 6.20 & 19.70 & 9.78 & 6.65 & 3.13 & 15.92 & 28.14 & 23.21 & 15.92 & 0.68 & 4.38 & 39.42 \\
\hline 5 & Number of leaf attached & $204.90 \pm 17.20$ & 30.67 & 671.67 & 12885.91 & 11998.52 & 887.39 & 14.54 & 55.40 & 53.46 & 14.54 & 0.93 & 217.74 & 106.27 \\
\hline 6 & Number of nodes & $28.27 \pm 3.10$ & 1.67 & 72.00 & 398.50 & 369.75 & 28.76 & 18.97 & 70.62 & 68.02 & 18.97 & 0.93 & 38.16 & 134.97 \\
\hline 7 & Total number of shoots & $5.13 \pm 0.56$ & 2.67 & 7.67 & 1.78 & 0.84 & 0.94 & 18.89 & 25.98 & 17.84 & 18.89 & 0.47 & 1.30 & 25.24 \\
\hline 8 & $\begin{array}{l}\text { Length of longest shoot } \\
\text { (cm) }\end{array}$ & $121.23 \pm 6.85$ & 74.33 & 167.33 & 717.72 & 576.93 & 140.79 & 9.79 & 22.10 & 19.81 & 9.79 & 0.80 & 44.36 & 36.59 \\
\hline 9 & Total shoot length $(\mathrm{cm})$ & $558.99 \pm 60.33$ & 250.33 & 977.67 & 44601.04 & 33681.73 & 10919.31 & 18.69 & 37.78 & 32.83 & 18.69 & 0.76 & 328.54 & 58.77 \\
\hline 10 & Leaf weight (gm) & $0.67 \pm 0.07$ & 0.09 & 1.44 & 0.14 & 0.13 & 0.02 & 18.46 & 56.47 & 53.37 & 18.46 & 0.89 & 0.70 & 103.90 \\
\hline 11 & Shoot weight (kg) & $1.06 \pm 0.13$ & 0.17 & 2.31 & 0.36 & 0.31 & 0.05 & 21.88 & 56.60 & 52.20 & 21.88 & 0.85 & 1.06 & 99.17 \\
\hline 12 & Total shoot weight (kg) & $3.40 \pm 0.35$ & 0.37 & 7.61 & 3.46 & 3.08 & 0.38 & 18.08 & 54.75 & 51.68 & 18.08 & 0.89 & 3.41 & 100.49 \\
\hline 13. & Leaf Yield (kg) & $3.05 \pm 0.42$ & 0.40 & 6.79 & 2.63 & 2.10 & 0.52 & 23.71 & 53.11 & 47.52 & 23.71 & 0.80 & 2.67 & 87.59 \\
\hline
\end{tabular}


Table.4 Estimate of genotypic and phenotypic correlation among yield attributing biometric traits in temperate mulberry germplasm accessions of Jammu and Kashmir

\begin{tabular}{|c|c|c|c|c|c|c|c|c|c|c|c|c|c|c|}
\hline Character & Level & $\begin{array}{l}\text { Petiole } \\
\text { Length } \\
(\mathrm{cm})\end{array}$ & $\begin{array}{l}\text { Petiole } \\
\text { Weight } \\
(\mathrm{gm})\end{array}$ & $\begin{array}{l}\text { Leaf } \\
\text { Length } \\
(\mathrm{cm})\end{array}$ & $\begin{array}{l}\text { Leaf } \\
\text { Width } \\
\text { (cm) }\end{array}$ & $\begin{array}{l}\text { Number } \\
\text { of leaf } \\
\text { attached }\end{array}$ & $\begin{array}{l}\text { Number } \\
\text { of nodes }\end{array}$ & $\begin{array}{l}\text { Total } \\
\text { number } \\
\text { of shoots }\end{array}$ & $\begin{array}{l}\text { Length of } \\
\text { longest } \\
\text { shoot } \\
(\mathrm{cm})\end{array}$ & $\begin{array}{l}\text { Total } \\
\text { Shoot } \\
\text { length } \\
(\mathrm{cm})\end{array}$ & $\begin{array}{l}\text { Leaf } \\
\text { Weight } \\
(\mathrm{kg})\end{array}$ & $\begin{array}{l}\text { Shoot } \\
\text { weight } \\
\text { (kg) }\end{array}$ & $\begin{array}{l}\text { Total } \\
\text { shoot } \\
\text { weight } \\
\text { (kg) }\end{array}$ & $\begin{array}{l}\text { Leaf } \\
\text { Yield } \\
(\mathrm{Kg})\end{array}$ \\
\hline $\begin{array}{l}\text { Petiole length } \\
(\mathrm{cm})\end{array}$ & $\begin{array}{l}\mathbf{G} \\
\mathbf{P}\end{array}$ & $\begin{array}{l}1.0000 \\
1.0000\end{array}$ & $\begin{array}{l}0.5311 * * \\
0.4430 * *\end{array}$ & $\begin{array}{l}0.5879 * * \\
0.5512 * *\end{array}$ & $\begin{array}{l}0.4573 * * \\
0.4580 * *\end{array}$ & $\begin{array}{r}-0.0113 \\
0.0030\end{array}$ & $\begin{array}{l}-0.0103 \\
-0.0030\end{array}$ & $\begin{array}{l}-0.0860 \\
-0.0398\end{array}$ & $\begin{array}{l}0.1602 \\
0.1139\end{array}$ & $\begin{array}{l}0.1385 \\
0.1143\end{array}$ & $\begin{array}{l}0.2831 * * \\
0.2207 * *\end{array}$ & $\begin{array}{l}0.2144^{*} \\
0.1729 *\end{array}$ & $\begin{array}{l}0.2132 \\
0.1522\end{array}$ & $\begin{array}{l}0.2461 * * \\
0.1696 * *\end{array}$ \\
\hline $\begin{array}{l}\text { Petiole weight } \\
\text { (gm) }\end{array}$ & $\begin{array}{l}\mathbf{G} \\
\mathbf{P}\end{array}$ & & $\begin{array}{l}1.0000 \\
1.0000\end{array}$ & $\begin{array}{l}0.6241 * * \\
0.5226 * *\end{array}$ & $\begin{array}{l}0.6236^{* *} \\
0.4441^{* *}\end{array}$ & $\begin{array}{l}-0.1626 \\
-0.1496\end{array}$ & $\begin{array}{l}-0.0257 \\
-0.0090\end{array}$ & $\begin{array}{l}-0.3118^{*} \\
-0.2041^{*}\end{array}$ & $\begin{array}{l}-0.0826 \\
-0.1199\end{array}$ & $\begin{array}{l}-0.1854 * \\
-0.1662 *\end{array}$ & $\begin{array}{l}0.1303 \\
0.1085\end{array}$ & $\begin{array}{l}-0.0286 \\
-0.0534\end{array}$ & $\begin{array}{l}-0.0269 \\
-0.0580\end{array}$ & $\begin{array}{l}0.0506 * * \\
0.0182 * *\end{array}$ \\
\hline $\begin{array}{l}\text { Leaf length } \\
(\mathrm{cm})\end{array}$ & $\begin{array}{l}\mathbf{G} \\
\mathbf{P}\end{array}$ & & & $\begin{array}{l}1.0000 \\
1.0000\end{array}$ & $\begin{array}{l}0.9286 * * \\
0.8307 * *\end{array}$ & $\begin{array}{l}-0.1407 \\
-0.1121\end{array}$ & $\begin{array}{l}-0.1370 \\
-0.1195\end{array}$ & $\begin{array}{l}-0.2670^{* *} \\
-0.2172 * *\end{array}$ & $\begin{array}{l}-0.0147 \\
-0.0361\end{array}$ & $\begin{array}{l}-0.0777 \\
-0.0937\end{array}$ & $\begin{array}{l}0.1485 \\
0.1166\end{array}$ & $\begin{array}{r}-0.0464 \\
0.0119\end{array}$ & $\begin{array}{l}0.0471 \\
0.0420\end{array}$ & $\begin{array}{l}0.0497 \\
0.0408\end{array}$ \\
\hline $\begin{array}{l}\text { Leaf width } \\
(\mathrm{cm})\end{array}$ & $\begin{array}{l}\mathbf{G} \\
\mathbf{P}\end{array}$ & & & & $\begin{array}{l}1.0000 \\
1.0000\end{array}$ & $\begin{array}{l}-0.0702 \\
-0.0487\end{array}$ & $\begin{array}{l}-0.1247 \\
-0.0901\end{array}$ & $\begin{array}{l}-0.2160 * \\
-0.1752^{*}\end{array}$ & $\begin{array}{l}-0.0523 \\
-0.0209\end{array}$ & $\begin{array}{l}-0.0743 \\
-0.0741\end{array}$ & $\begin{array}{l}0.2378^{*} \\
0.1737^{*}\end{array}$ & $\begin{array}{l}0.0086 \\
0.0453\end{array}$ & $\begin{array}{l}0.1026 \\
0.0885\end{array}$ & $\begin{array}{l}0.1400 \\
0.0855\end{array}$ \\
\hline $\begin{array}{l}\text { Number of } \\
\text { leaf attached }\end{array}$ & $\begin{array}{l}\mathbf{G} \\
\mathbf{P}\end{array}$ & & & & & $\begin{array}{l}1.0000 \\
1.0000\end{array}$ & $\begin{array}{l}-0.2468 * * \\
-0.2256 * *\end{array}$ & $\begin{array}{l}0.0822 \\
0.0354\end{array}$ & $\begin{array}{l}0.5948 * * \\
0.5391 * *\end{array}$ & $\begin{array}{l}0.3899 * * \\
0.3293 * *\end{array}$ & $\begin{array}{l}0.6749 * * \\
0.6622 * *\end{array}$ & $\begin{array}{l}0.7462 * * \\
0.6967 * *\end{array}$ & $\begin{array}{l}0.5753 * * \\
0.5448 * *\end{array}$ & $\begin{array}{l}0.6346 * * \\
0.5819 * *\end{array}$ \\
\hline $\begin{array}{l}\text { Number of } \\
\text { nodes }\end{array}$ & $\begin{array}{l}\mathbf{G} \\
\mathbf{P}\end{array}$ & & & & & & $\begin{array}{l}1.0000 \\
1.0000\end{array}$ & $\begin{array}{l}0.1298 \\
0.0810\end{array}$ & $\begin{array}{l}0.1142 \\
0.1091\end{array}$ & $\begin{array}{l}0.1335 \\
0.1135\end{array}$ & $\begin{array}{l}0.1051 \\
0.1051\end{array}$ & $\begin{array}{r}-0.0052 \\
0.0143\end{array}$ & $\begin{array}{l}-0.1337 \\
-0.1009\end{array}$ & $\begin{array}{l}0.0074 \\
0.0178\end{array}$ \\
\hline $\begin{array}{l}\text { Total number } \\
\text { of shoots }\end{array}$ & $\begin{array}{l}\mathbf{G} \\
\mathbf{P}\end{array}$ & & & & & & & $\begin{array}{l}1.0000 \\
1.0000\end{array}$ & $\begin{array}{l}0.4135 \\
0.3520\end{array}$ & $\begin{array}{l}0.7918 \\
0.8008\end{array}$ & $\begin{array}{l}0.1038 \\
0.0454\end{array}$ & $\begin{array}{l}0.2634 \\
0.1530\end{array}$ & $\begin{array}{l}0.5883 \\
0.4429\end{array}$ & $\begin{array}{l}0.5271 * * \\
0.4457 * *\end{array}$ \\
\hline $\begin{array}{l}\text { Length of } \\
\text { longest shoot } \\
(\mathrm{cm})\end{array}$ & $\begin{array}{l}\mathbf{G} \\
\mathbf{P}\end{array}$ & & & & & & & & $\begin{array}{l}1.0000 \\
1.0000\end{array}$ & $\begin{array}{l}0.8640 * * \\
0.7782 * *\end{array}$ & $\begin{array}{l}0.6333 * * \\
0.5817 * *\end{array}$ & $\begin{array}{l}0.8367 * * \\
0.7427 * *\end{array}$ & $\begin{array}{l}0.8323 * * \\
0.7777 * *\end{array}$ & $\begin{array}{l}0.8311 * * \\
0.7442 * *\end{array}$ \\
\hline $\begin{array}{l}\text { Total shoot } \\
\text { length }(\mathrm{cm})\end{array}$ & $\begin{array}{l}\mathbf{G} \\
\mathbf{P}\end{array}$ & & & & & & & & & $\begin{array}{l}1.0000 \\
1.0000\end{array}$ & $\begin{array}{l}0.4225 * * \\
0.3423 * *\end{array}$ & $\begin{array}{l}0.6389 * * \\
0.5210 * *\end{array}$ & $\begin{array}{l}0.8623 * * \\
0.7765 * *\end{array}$ & $\begin{array}{l}0.8167 * * \\
0.7461 * *\end{array}$ \\
\hline $\begin{array}{l}\text { Leaf weight } \\
\text { (gm) }\end{array}$ & $\begin{array}{l}\mathbf{G} \\
\mathbf{P}\end{array}$ & & & & & & & & & & $\begin{array}{l}1.0000 \\
1.0000\end{array}$ & $\begin{array}{l}0.8515^{* *} \\
0.8108^{* *}\end{array}$ & $\begin{array}{l}0.6660 * * \\
0.6282 * *\end{array}$ & $\begin{array}{l}0.8074 * * \\
0.7442 * *\end{array}$ \\
\hline $\begin{array}{l}\text { Shoot weight } \\
\text { (kg) }\end{array}$ & $\begin{array}{l}\mathbf{G} \\
\mathbf{P}\end{array}$ & & & & & & & & & & & $\begin{array}{l}1.0000 \\
1.0000\end{array}$ & $\begin{array}{l}0.8449 * * \\
0.7951 * *\end{array}$ & $\begin{array}{l}0.8630 * * \\
0.7771 * *\end{array}$ \\
\hline $\begin{array}{l}\text { Total shoot } \\
\text { weight }(\mathrm{kg})\end{array}$ & $\begin{array}{l}\mathbf{G} \\
\mathbf{P}\end{array}$ & & & & & & & & & & & & $\begin{array}{l}1.0000 \\
1.0000\end{array}$ & $\begin{array}{l}0.9404 * * \\
0.8975 * *\end{array}$ \\
\hline
\end{tabular}


Table.5 Direct (diagnol) and indirect effects of component characters contributing to total leaf weight in in temperate mulberry germplasm accessions of Jammu and Kashmir

\begin{tabular}{|c|c|c|c|c|c|c|c|c|c|c|c|c|c|c|}
\hline Character & Level & $\begin{array}{l}\text { Petiole } \\
\text { Length } \\
(\mathrm{cm})\end{array}$ & $\begin{array}{l}\text { Petiole } \\
\text { Weight } \\
(\mathrm{gm})\end{array}$ & $\begin{array}{l}\text { Leaf } \\
\text { Length } \\
(\mathrm{cm})\end{array}$ & $\begin{array}{l}\text { Leaf } \\
\text { Width } \\
(\mathrm{cm})\end{array}$ & $\begin{array}{l}\text { Number } \\
\text { of leaf } \\
\text { attached }\end{array}$ & $\begin{array}{l}\text { Number } \\
\text { of nodes }\end{array}$ & $\begin{array}{l}\text { Total } \\
\text { number } \\
\text { of } \\
\text { shoots }\end{array}$ & $\begin{array}{l}\text { Length } \\
\text { of } \\
\text { longest } \\
\text { shoot } \\
\text { (cm) }\end{array}$ & $\begin{array}{l}\text { Total } \\
\text { Shoot } \\
\text { length } \\
(\mathrm{cm})\end{array}$ & $\begin{array}{l}\text { Leaf } \\
\text { Weight } \\
(\mathrm{kg})\end{array}$ & $\begin{array}{l}\text { Shoot } \\
\text { weight } \\
(\mathrm{kg})\end{array}$ & $\begin{array}{l}\text { Total } \\
\text { shoot } \\
\text { weight } \\
\text { (kg) }\end{array}$ & $\begin{array}{l}\text { Leaf } \\
\text { Yield } \\
(\mathrm{Kg})\end{array}$ \\
\hline \multirow[t]{2}{*}{ Petiole length (cm) } & G & 0.0016 & 0.0009 & 0.0009 & 0.0007 & 0.0000 & 0.0000 & -0.0001 & 0.0003 & 0.0002 & 0.0005 & 0.0003 & 0.0003 & 0.2461 \\
\hline & $\mathrm{P}$ & -0.0369 & -0.0163 & -0.0203 & -0.0169 & -0.0001 & 0.0001 & 0.0015 & -0.0042 & -0.0042 & -0.0081 & -0.0064 & -0.0056 & -0.0063 \\
\hline \multirow[t]{2}{*}{ Petiole weight (gm) } & G & 0.0652 & 0.1228 & 0.0766 & 0.0766 & -0.0200 & -0.0032 & -0.0383 & -0.0101 & -0.0228 & 0.0160 & -0.0035 & -0.0033 & 0.0506 \\
\hline & $\mathrm{P}$ & 0.0319 & 0.0721 & 0.0377 & 0.0320 & -0.0108 & -0.0006 & -0.0147 & -0.0086 & -0.0120 & 0.0078 & -0.0038 & -0.0042 & 0.0013 \\
\hline \multirow[t]{2}{*}{ Leaf length $(\mathrm{cm})$} & G & -0.1488 & -0.1580 & -0.2532 & -0.2351 & 0.0356 & 0.0347 & 0.0676 & 0.0037 & 0.0197 & -0.0376 & 0.0117 & -0.0119 & 0.0498 \\
\hline & $\mathrm{P}$ & 0.0036 & 0.0034 & 0.0066 & 0.0055 & -0.0007 & -0.0008 & -0.0014 & -0.0002 & -0.0006 & 0.0008 & 0.0001 & 0.0003 & 0.0003 \\
\hline \multirow[t]{2}{*}{ Leaf width (cm) } & G & 0.0674 & 0.0919 & 0.1369 & 0.1474 & -0.0104 & -0.0184 & -0.0318 & -0.0077 & -0.0109 & 0.0350 & 0.0013 & 0.0151 & 0.1400 \\
\hline & $\mathrm{P}$ & -0.0110 & -0.0106 & -0.0199 & -0.0239 & 0.0012 & 0.0022 & 0.0042 & 0.0005 & 0.0018 & -0.0042 & -0.0011 & -0.0021 & -0.0020 \\
\hline \multirow[t]{2}{*}{ Number of leaf attached } & G & $-0.0005-$ & -0.0067 & -0.0058 & -0.0029 & 0.0410 & -0.0101 & 0.0034 & 0.0244 & 0.0160 & 0.0277 & 0.0306 & 0.0236 & -0.6346 \\
\hline & $\mathrm{P}$ & 0.0002 & -0.0077 & -0.0057 & -0.0025 & 0.0512 & -0.0116 & -0.0018 & 0.0276 & 0.0169 & 0.0339 & 0.0357 & 0.0279 & 0.0298 \\
\hline \multirow[t]{2}{*}{ Number of nodes } & G & 0.0004 & 0.0011 & 0.0058 & 0.0053 & 0.0104 & -0.0422 & -0.0055 & -0.0048 & -0.0056 & -0.0044 & 0.0002 & 0.0056 & 0.0074 \\
\hline & $\mathrm{P}$ & -0.0001 & -0.0002 & -0.0020 & -0.0015 & -0.0039 & 0.0171 & 0.0014 & 0.0019 & 0.0019 & 0.0018 & 0.0002 & -0.0017 & -0.0003 \\
\hline \multirow[t]{2}{*}{ Total number of shoots } & G & 0.0106 & 0.0386 & 0.0330 & 0.0267 & -0.0102 & -0.0161 & 0.1238 & -0.0512 & -0.0980 & -0.0128 & -0.0326 & -0.0728 & 0.5271 \\
\hline & $P$ & 0.0011 & 0.0058 & 0.0062 & 0.0050 & -0.0010 & -0.0023 & -0.0286 & -0.0101 & -0.0229 & -0.0013 & -0.0044 & -0.0127 & -0.0128 \\
\hline \multirow{2}{*}{$\begin{array}{l}\text { Length of longest shoot } \\
(\mathrm{cm})\end{array}$} & G & -0.0255 & 0.0132 & 0.0023 & 0.0083 & -0.0948 & -0.0182 & -0.0659 & -0.1593 & -0.1377 & -0.1009 & -0.1333 & -0.1326 & 0.8311 \\
\hline & $P$ & -0.0138 & 0.0146 & 0.0044 & 0.0025 & -0.0654 & -0.0132 & -0.0427 & -0.1213 & -0.0944 & -0.0706 & -0.0901 & -0.0944 & -0.0903 \\
\hline \multirow[t]{2}{*}{ Total shoot length $(\mathrm{cm})$} & G & 0.0836 & -0.1119 & -0.0469 & -0.0448 & 0.2354 & 0.0806 & 0.4781 & 0.5217 & 0.6038 & 0.2551 & 0.3858 & 0.5207 & 0.8167 \\
\hline & $\mathrm{P}$ & 0.0405 & -0.0589 & -0.0332 & -0.0263 & 0.1167 & 0.0402 & 0.2837 & 0.2757 & 0.3543 & 0.1213 & 0.1846 & 0.2751 & 0.2643 \\
\hline \multirow[t]{2}{*}{ Leaf weight (gm) } & G & 0.1411 & 0.0649 & 0.0740 & 0.1185 & 0.3364 & 0.0524 & 0.0517 & 0.3156 & 0.2106 & 0.4984 & 0.4244 & 0.3319 & 0.8074 \\
\hline & $\mathrm{P}$ & 0.0867 & 0.0426 & 0.0458 & 0.0682 & 0.2601 & 0.0413 & 0.0178 & 0.2285 & 0.1344 & 0.3928 & 0.3184 & 0.2467 & 0.2923 \\
\hline \multirow[t]{2}{*}{ Shoot weight (kg) } & G & -0.0335 & 0.0045 & 0.0073 & -0.0013 & -0.1167 & 0.0008 & -0.0412 & -0.1308 & -0.0999 & -0.1332 & -0.1564 & -0.1321 & 0.8630 \\
\hline & $\mathrm{P}$ & -0.0133 & 0.0041 & -0.0009 & -0.0035 & -0.0537 & -0.0011 & -0.0018 & -0.0573 & -0.0402 & -0.0625 & -0.0771 & -0.0613 & -0.0599 \\
\hline \multirow[t]{2}{*}{ Total shoot weight (kg) } & G & 0.0844 & -0.0107 & 0.0187 & 0.0406 & 0.2278 & -0.0529 & 0.2329 & 0.3295 & 0.3414 & 0.2637 & 0.3345 & 0.3960 & 0.9404 \\
\hline & $\mathrm{P}$ & 0.0806 & -0.0307 & 0.0223 & 0.0469 & 0.2885 & -0.0534 & 0.2346 & 0.4118 & 0.4112 & 0.3327 & 0.4210 & 0.5296 & 0.4753 \\
\hline
\end{tabular}


Fig.1 Coefficient of variations (ECV, PCV and GCV), heritability (Broad Sense) and genetic advancement $5 \%$ for 47 mulberry genotypes for agro-morphological markers

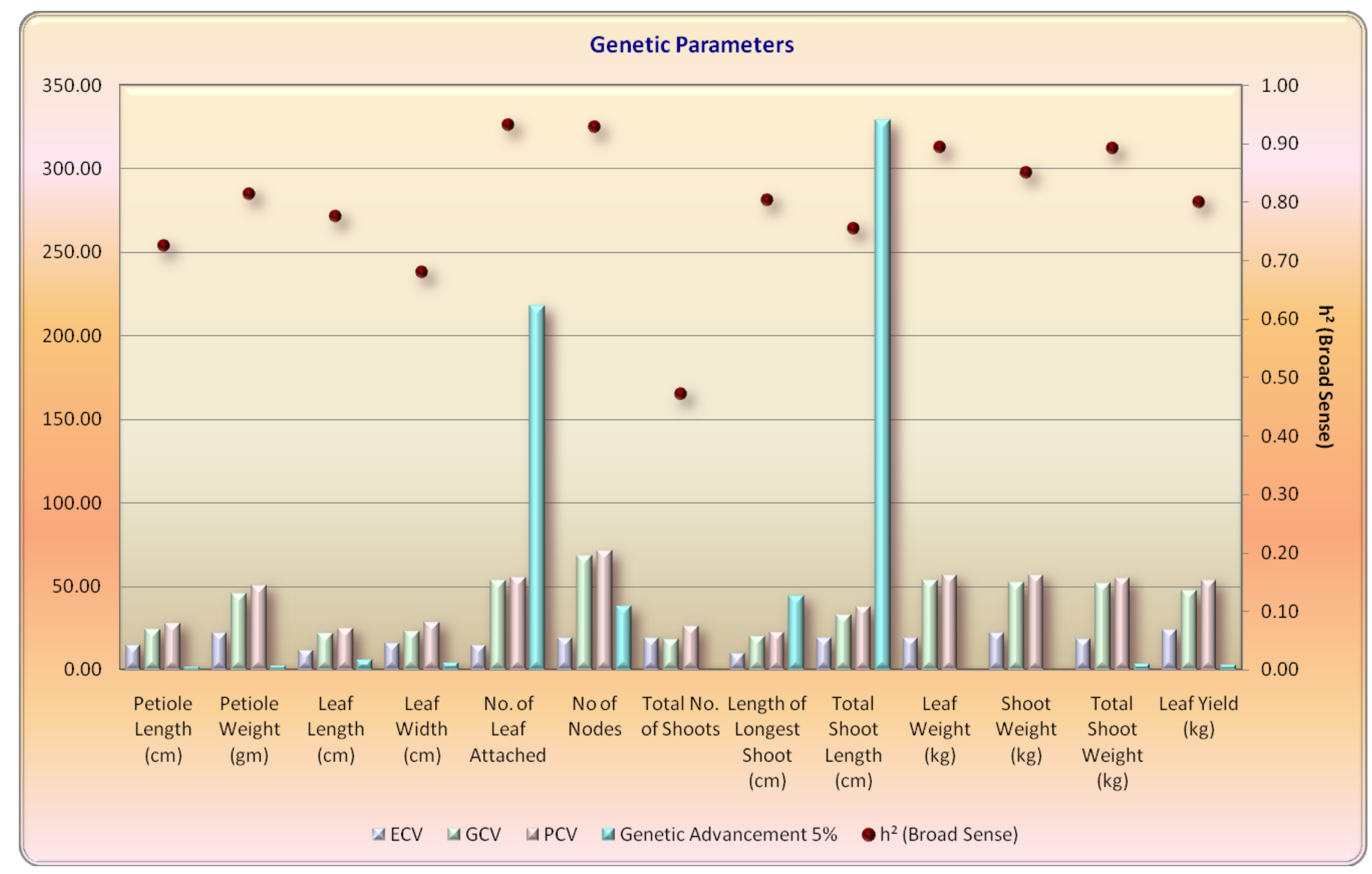


So it is necessary to determine the path coefficients which partition the observed correlation into direct and indirect effects and also ravels the cause and effect relationship between yield and their related traits. By partitioning the phenotypic and genotypic correlations, the direct effect of a chosen trait on leaf yield and its indirect effect through other characters were computed (Table 5).

The path coefficient estimates indicated that total shoot length had the highest positive direct effect (0.6038) followed by leaf weight (0.4984), total shoot weight $(0.3960)$, leaf width (0.1474), total number of shoots (0.1238), petiole weight $(0.1228)$, number of leaf attached (0.0410) and petiole length (0.0016) indicated that selection for these traits would improve leaf yield. Leaf length ($0.2532)$, length of longest shoot (-0.1593), shoot weight (-0.1571) and number of nodes $(-0.0 .422)$ showed negative direct effect on leaf yield. Similar results were obtained by Banerjee et al., (2007), Doss et al., (2012) and Suresh et al., (2017).

The results of path analysis from germplasm lines also indicated high positive indirect effect of total shoot length $(0.5207$ and 0.4781 ) had highest positive indirect effect on leaf yield via total shoot weight and total number of shoots. Considering the overall direct and indirect effects of various growth parameters on leaf yield in mulberry, total number of shoots, total shoot weight, total shoot length may be the most valuable characters of mulberry for the selection programme.

The present study indicated that there is adequate genetic variability present in the genotypes studied. Based on the studies on genetic variability parameters (broad sense heritability, genetic advance) and trait association (Correlation and Path analysis) it is concluded that length of longest shoot, total number of shoots, total shoot length, shoot weight, leaf weight and total shoot weight were the most important yield attributing components. A wide spectrum of genetic variability among the genotypes indicated the possibilities of improvement in leaf yield through successful breeding programmes.

\section{References}

Ahsan, M. M., Dhar, A., Dhar, K. L. and Fotadar, R. K. 1990. Package of practices for mulberry cultivation under temperate conditions. Indian Silk, 29(2):7-12.

Al-Aysh, F., Kutma, H., Serhan, M., AlZoubai, A. and Al-Naseer, M. A. 2012. Genetic analysis and correlation studies of yield and fruit quality traits in tomato (Solanum lycopersicum L.). New York Sci. J., 5(10):142-145.

Al-Jibourie, H. A., Miller, P. A. and Robinson, H. F. 1958. Genotypic and environmental variance in an upland cotton crosses of interspecific origin. Agron. J., 50:663-637.

Banerjee, R., Roychowdhuri, S., Sau, H., Das, B. K., Ghosh, Pannalal and Saratchandra, B., 2007. Genetic diversity and interrelationship among mulberry genotypes. J. Genet. Genomes, 34(8):691-697.

Bari, M. A., Qaiyyum, M. A. and Ahmed, S. U., 1989. Correlation studies in mulberry (Morus alba L.) Indian $J$. Seric., 258(1):11-16.

Benavides, J. E., Lachaux, M. and Fuentes, M. 1994. Effect of goat manure application in soil on biomass production and quality of mulberry (Morus sp.). In: Benavides J.E. (ed.): Trees and Shrubs in Central America. Volume II. CATIE, Turrialba, 495-514. (in Spanish).

Bhatt, G.M. 1973. Significance of path coefficient analysis in determining the 
nature of character association. Euphytica, 22:338-343.

Biradar, J., Chikkalingaiah, Banuprakash, K. G., Narayanaswamy, T. K. and Bhaskar, R. N., 2015. Estimation of genetic variability and correlation studies in mulberry Morus spp. Mysore J. Agric., Sci., 49(3):516-522.

Burton, G. W. and De Vane, E. H., 1953. Estimating heritability in tall Festuca (Festuca arundinaceae) from replicated clonal material. Agron. J., 45: 478-481.

Chikkalingaiah, Y. B., Vijayashekara, T., Geetha Devi and Sadatulla, F., 2009.Correlation and path co-efficient analysis in different accessions of mulberry. In: Proc. National Workshop on Seri-Biodiversity Conservation, 7-8 March, 2009. pp. 54-58.

Clewer, A. G. and Scarisbrick, D. H., 2001. Practical statistics and experimental design for plant and crop science (p. 346). John Wiley and Sons Ltd., New York.

Dar, R. A., Sharma, J. P., Gupta, R. K. and Chopra, S. 2011. Studies on correlation and path analysis for yield andphysicchemical traits in tomato (Lycopersicon esculentum Mill). Vegetos, 24(2): 136141.

Das, B. C. and Krishnaswamy, S., 1969.Estimation of components of variation of leaf yield and its related traits in mulberry. J. Seric. Sci. Japan., 38(3):242-248.

Dewey, D. R. and Lu, K. H., 1959. A correlation and path coefficient analysis of components of -crested wheatgrass seed production. Agron, J., 51: 515-518. http://dx.doi.org/10.2134/agronj1959.00 021962005100090002x.

Doss, S. G., Chakraborti, S. P., Roychowdhuri, S., Das, N. K., Vijayan, K., Ghosh, P. D., Rajan, M. V. and Qadri, S. M. H. 2012. Variability, heritability and genetic advance in mulberry (Morus spp.) for growth and yield attributes. Agric. Sci., 3(2):2018213. doi:10.4236/as.2012.32024.

Doss, S. G., Chakraborti, S. P., Roychowdhuri, S., Das, N. K., Vijayan, K. and Ghosh, P. D. 2011. Character association in improved mulberry genotypes exhibiting delayed leaf senescence. J. Ornamental Hortic. Plants, 1(2): 85-95.

Doss, S.G., Rahman, M.S., Debnath, S., Ghosh, M.K., Sau, H., Ghosh, P. L. and Sarkar, A., 2006.Variability, heritability and genetic advance in nine germplasm lines of mulberry (Morus spp.). Indian J. Genet., 66, 169-170.

Goel, A. K., Ravindran, S., Rao, A. A., Naik, G. V., Tikader, A., Mukherjee, P. and Sekar, S., 1998. Variability in rooting parameters at juvenile stage in mulberry (Morus spp.) germplasm. Indian $J$. Seric., 37(2):109-112.

Golani, I. J., Mehta, D. R., Purohit, V. L., Pandya, H. M. and Kanzariya, M. V. 2007. Genetic variability, correlation and path coefficient studies in tomato. Indian J. Agric. Res., 41(2):146-149.

Harer, P. N., Lad, D. B. and Bhor, T. J. 2003. Correlation and path analysis studies in tomato. J. Maharashtra Agric. Universities, 27(3): 302-303.

Islam, B. M. R., Ivy, N. A., Rasul, M. G. and Zakaria, M. 2010. Character association and path analysis of exotic tomato (Solanum lycopersicum L.) genotypes. Bangladesh J. Plant Breed. Genet., 23(1):13-18.

Johnson, H. W., Robinson, H. F. and Comstock, R. E. 1955. Genotypic and phenotypic correlations in soybean and their implications in selection. Agron J., 47: 477-483.

Keshava Murthy, B. C., Puttaraju, H. P. and Hittalmani, S. 2010. Genetic variability and correlation studies in selected mulberry (Morus spp.) germplasm 
accessions. Elect. J. Plant Breed., 1(3): 351-355.

Liang, G. H. and Walter, T. L. 1968. Heritability estimates and gene effects for agronomic trials in grain sorghum. Crop Sci., 8(1):77-80.

Lush, J. L., 1945. Heritability of quantitative characters in farm animals. In: Proc. $8^{\text {th }}$ Congr. Genet. Heriditas, 35:356-375.

Mallikarjunappa, R. S., Venkateshaiah, H. V., Rao, M. S. E., Anantharaman, M. N. and Bongale, U. D. 2008. Genetic variability and correlation studies in mulberry germplasm. Indian J. Seric., 47(2):226-229.

Masilamani, S., Reddy, A.R., Sarkar, A., Srinivas, B.T. and Kamble, C. K. 2000. Heritability and genetic advance of quantitative traits in mulberry (Morus spp.). Indian J. Seric., 39, 16-20.

Panse, V. G. 1957. Genetics of quantitative characters in relation to plant breeding. Indian J. Genet., 17(3):318-328.

Panse, V.G. and Sukhatme, P.V. 1985. Statistical Methods for Agricultural Workers (2nd ed., p. 381). Indian Council of Agricultural Research, New Delhi.

Rahman, M.S., Doss, S.G., Debnath, S., Roychowdhuri, S., Ghosh, P. L. and Sarkar, A. 2006. Genetic variability and correlation studies of leaf characters in some mul-berry (Morus spp.) germplasm accessions. Indian J. Genet., 66:359-360.

Sarkar, A., Roy, B. N., Gupta, K. K. and Das, B. C., 1987. Character association in mulberry under close planting. Indian $J$. Seric., 25(2):76-78.

Sivaprasad, V., Naik, G. V., Doss, S. G., Rajashekar, K. and Thippeswamy, T., 2016. Guidelines for the conduct of test for Distinctiveness, Uniformity and Stability on Mulberry (Morus spp.). pp. $1-20$.
Souza, L. M., Melo, P. C. T., Luders, R. R. and Melo, A. M. T. 2012. Correlations between yield and fruit quality characteristics of fresh market tomatoes. Horticultura Brasileira, 30: 627-631. http://dx.doi.org/10.1590/S0102-05362 012000400011.

Suresh, K., Jalaja, S. K., Banerjee, R. and Trivedy, K., 2017. Genetic variability, correlation and path analysis in physiological and yield attributes in mulberry (Morus spp.). J. Crop Weed, 13(1):28-33.

Susheelamma, B.N., Jolly, M.S., Giridhar, K.S., Dwivedi, N.K. and Suryanarayana, N. 1998. Correlation and path analysis in mulberry under stress and non-stress conditions. Sericologia, 28:239-244.

Tanksley, S. D. and McCouch, S. R. 1997. Seed banks and molecular maps: unlocking genetic potential from the wild. Science, 277:1063-1066.

Tasisa, J., Belew, D. and Bantte, K. 2012. Genetic association analysis among some traits of tomato (Lycopersicon esculentum Mill.) genotypes in West Showa, Ethiopia. Int. J. Plant Breed. Genet., 6(3): 129-139. http://dx.doi.org/ 10.3923/ijpbg.2012.129.139

Tikadar, A., 1997. Studies on heritabilities, genetic parameters and response to selection in mulberry. Bull. Seric. Res., 8:19-22.

Tikader, A. and Dandin, S. B., 2005. Evaluation of Morusserrata Roxb. Mulberry germplasm in ex-situ field gene bank. Indian J. Seric., 44(1):45-49.

Tikader, A. and Kamble, C. K., 2008a. Studies on variability of indigenous mulberry germplasm on growth and leaf yield. Pertanika J. Trop. Agric. Sci., 31:163-170.

Tikader, A. and Roy, B. N., 1999. Genetic variability and character association in 
mulberry (Morus spp.) germplasm accessions. Indian J. For., 22:26-29.

Tikader, A. and Roy, B. N., 2001. Multivariate analysis in some mulberry (Morus spp.) germplasm accessions. Indian J. Seric., 40(2):168-170.

Tikader, A., Thangavelu, K. and Rao, A. A., 2004. Characterization and evaluation of mulberry (Morus spp.) germplasm. Indian J. Seric., 43(1):106-110.

Tiwari, J. K. and Upadhyay, D. 2011. Correlation and path-coefficient studies in tomato (Lycopersicon esculentum Mill.). Res. J. Agric. Sci., 2(1):63-68.

Vijayan, K., Chakraborti, S. P., Doss, S. G., Tikader, A. and Roy, B. N., 1998. Evaluation of triploid mulberry genotypes 1. Morphological and anatomical studies. Indian J. Seric. 37(1):64-67.

Vijayan, K., Doss, S. G., Chackraborti, S. P., Ghosh, P. D. and Saratchandra, B.,
2010. Character association in mulberry under different magnitude of salinity stress. Emir. J. Food Agric., 22(4): 318325.

Vijayan, K., Tikader, A., Das, K. K., Chakraborti, S. P. and Roy, B. N., 1997b. Correlation studies in mulberry (Morus spp.). Indian J. Genet. Breed., 57:455-460.

Vijayashekara, Y. B., 2009. Genetic variation and character association in different accessions of mulberry (Morus sp.). M.Sc. Thesis, University of Agricultural Sciences, Bangalore. pp. 1-161.

Wright, S., 1921.Correlation and causation. $J$. Agric. Res., 20:557-585.

Zhao, W. G., Miao, X. X., Zang, B., Zhang, L., Pan, Y. L. and Huang, Y. P., 2006. Construction of fingerprinting and genetic diversity of mulberry cultivars in China by ISSR markers. Acta Genet. Sin, 33:851-860.

\section{How to cite this article:}

Pawan Saini, S.S. Chauhan, Aftab A. Shabnam, Lal Chand and Narender Negi . 2018. Genetic Variability and Trait Association Analysis for Agro-Morphological Markers in Mulberry Genetic Resources from Kashmir, India. Int.J.Curr.Microbiol.App.Sci. 7(04): 1799-1812. doi: https://doi.org/10.20546/ijcmas.2018.704.204 